\title{
La presencia social en ambientes virtuales de aprendizaje: una estrategia de interacción y construcción del conocimiento'
}

\author{
Martha Liliana Palomino Leiva ${ }^{2}$ \\ Gabriel Mauricio Ramírez Villegas ${ }^{3}$
}

\begin{abstract}
Resumen
El presente artículo busca comprender la presencia social de un entorno de aprendizaje en línea soportado en la plataforma Moodle. Este concepto iniciado por Gunawardena en 1995 explica el grado en que las personas se perciben de forma real en un ambiente de aprendizaje mediado por computador (CMC). Por ello, se buscó describir las interacciones de tutores y estudiantes dadas en los foros colaborativos de un aula virtual Moodle, además de revisar las estrategias de comunicación, las relaciones establecidas y la motivación por el aprendizaje. Este abordaje se desarrolló a partir de los estudios desarrollados por Rourke, Anderson, Garrison \& Archer (2001), Tu \& McIsaac (2002), Espinoza, et al. (2008).
\end{abstract}

Este trabajo se desarrolló empleando la encuesta y la observación como instrumentos de indagación. Entre los resultados se destaca la importancia de las estrategias afectivas e interactivas para el desarrollo de la presencia social en el curso. De estas estrategias se identifican la identidad personal, los saludos de bienvenida, la participación, y la motivación entre los miembros de los grupos colaborativos y el tutor.

Estas contribuyen a establecer lazos de amistad y de cooperación entre los mismos pero poco propician la intimidad y la cercanía entre

\footnotetext{
1 Esta es una investigación como opción de grado de los estudiantes Martha Liliana Palomino Leiva y Gabriel Mauricio Ramírez Villegas, del programa Master of Arts in Education- Specialization in Online Education. National Distance Education University - UNAD Florida.

2 Tutora programa Psicología. Escuela Ciencias Sociales, Artes y Humanidades. Universidad Nacional Abierta y a Distancia unad. CERES Santander de Quilichao. E-mail: martha.palomino@unad.edu.co.

3 Tutor Programa Ingeniería de Sistemas. Escuela Ciencias Básicas, Tecnologías e Ingeniería. Universidad Nacional Abierta y a Distancia, UNAD. CEAD Palmira E-mail: gabriel.ramirez@unad.edu.co.
} 
los participantes. Por último, este trabajo se realizó en la Universidad Nacional Abierta y a Distancia, UNAD - Colombia, en el curso virtual de investigación cualitativa durante el primer periodo académico de 2010.

Palabras clave: presencia social, constructivismo, interacción, aprendizaje colaborativo.

\title{
Social presence in virtual learning environments: a strategy of interaction and knowledge construction
}

\begin{abstract}
This article seeks to understand the social presence of an online learning environment supported by Moodle platform. This concept started in 1995 by Gunawardena who explains the extent to which people perceive real form in a computer-mediated learning (CMC). Therefore, we tried to describe the interactions given by the tutors and students in collaborative forums Moodle virtual classroom, in addition to review communication strategies, the relationships established and motivation for learning. This approach was developed from the studies by Rourke, Anderson, Garrison \& Archer (2001), Tu \& McIsaac (2002), Espinoza, et al. (2008). This work was developed from survey and observation as research instruments. The results highlight the importance of affective and interactive strategies for the development of social presence on the course. From these strategies it is identified personal identity, the greetings of welcome, participation and motivation among the members of collaborative groups and the tutor. These strategies help to establish bonds of friendship and cooperation between them but they are not favorable to intimacy and closeness among participants. Finally, this study was conducted at the National Open University at Distance, UNAD - Colombia, in qualitative research online course during the first academic term in 2010 .
\end{abstract}

Key words: social presence, constructivism, interaction, collaborative learning. 


\section{Introducción}

La educación en línea es un modelo educativo que utiliza internet para el desarrollo del proceso de enseñanza-aprendizaje e implica un proceso formativo mediado que permite la interacción en doble vía en forma asincrónica y sincrónica. Su modelo pedagógico se centra en el aprendizaje autónomo y significativo, implementado desde la perspectiva del constructivismo social, que refleja la construcción del conocimiento a través de la interacción de los que aprenden.

Desde esta perspectiva, la interacción - construida dentro de un ambiente virtual o en línea y bajo un modelo formativo permanente - logra conformar comunidades de aprendizaje (McConnell, 2000; Palloff \& Pratt, 1999). Esta estrategia educativa permite crear ambientes dinámicos, flexibles y críticos centrados en el aprendizaje colaborativo. En el contexto de las comunidades de aprendizaje y con el propósito de apoyar el pensamiento crítico de quienes utilizan la Comunicación Mediada por computador (CMC), Garrison (2000) — citado por Jerónimo (2009) — destaca la importancia de aprender en línea vinculando tres componentes: la presencia cognitiva, el docente y lo social.

La presencia cognitiva es definida por Garrison et al. (2000) como "el grado al cual los participantes son capaces de construir el significado a partir de una comunicación sostenida". Desde la perspectiva de Anderson, Garrison \& Rourke (2001), la presencia docente incluye el diseño educativo y la conducción de los procesos de aprendizaje, en los que el docente aporta la maestría del tema como experto y facilita un aprendizaje activo, en tanto que la presencia social tiene como función apoyar los objetivos cognoscitivos y afectivos del aprendizaje.

Jerónimo (2009) define la presencia social como la capacidad de los principiantes de proyectarse social y emocionalmente en una comunidad de indagación. Se apoya el cumplimiento de objetivos afectivos haciendo que en las interacciones del grupo se rescaten y den continuidad a los aportes de los integrantes, siempre y cuando se hagan de manera frecuente. Así se recompensa de manera intrínseca la pertenencia a la comunidad, conduciendo las actividades hacia el incremento de la integración académica, social e institucional. Esta integración posibilita la perseverancia del estudiante en el curso académico.

La presencia social en la educación a distancia y en línea está relacionada con diferentes factores y necesidades básicas del ser humano, que requiere de algún tipo de contacto a fin de realizar las actividades educativas. De acuerdo con diversos autores, la presencia social es un tema complejo que debe ser estudiado de diversas formas, teniendo en cuenta factores como la comunicación, la interacción y el aprendizaje.

Siguiendo esta discusión, Gunawardena (1995) define la presencia social como el grado en que las personas se perciben de forma real en un aprendizaje mediado 
por computadores (CMC). Tu \& McIsaac (2002) la señalan como "el grado de sentimiento, la percepción y la reacción de estar conectado por CMC" a otra persona (p. 140). Picciano (2002) expresa que la presencia social es como la percepción del estudiante de estar en y de pertenecer a un curso en línea.

Se entiende la presencia social como el grado en que se percibe a una persona en el resultado de las interacciones personales en la comunicación mediada (Gunawardena, 2003). En lo que respecta al curso en línea, éste tiene la capacidad de transmitir algunas comunicaciones en dos vías que propician la intimidad y cercanía para establecer un clima de confianza entre los participantes, de modo que se sientan parte de la comunidad de aprendizaje.

Short et al. (1976) definen la presencia social como el grado de relevancia (es decir, la calidad o el hecho de estar allí) entre dos comunicadores, utilizando un medio de comunicación. Según esto, la presencia social es un atributo fundamental de los medios de comunicación que puede determinar la forma en que las personas interactúan y se comunican. Además, la percepción que se tiene de las personas que aparecen en algunos medios de comunicación proyecta un mayor grado de presencia social (por ejemplo el vídeo, que incluye audio e imagen como una manera ilimitada de comunicación).

Al interactuar por este medio, el estudiante puede tener la inmediatez necesaria para sentirse cercano y próximo a sus compañeros. Esto puede "humanizar" el clima a través de sus acercamientos empáticos, al responder rápidamente las comunicaciones, propiciando la revelación de algunas dimensiones "humanas" de la propia personalidad y, en general, el respeto a las personas.

La teoría de la presencia social ha sido usada para valorar las reacciones interpersonales en la comunicación mediada por computadora, donde la ausencia de comunicación no verbal podría suponer dificultades en las interacciones; sin embargo, las investigaciones reportan que existe la posibilidad de desarrollar "comunidades en línea" con comportamientos positivos y relaciones cálidas y amistosas. Dos conceptos se asocian con la presencia social: la intimidad y la inmediatez. Este último contribuye a la intimidad en la comunicación mediada por computadora. (Gunawardena, 2003).

La presencia social efectiva predice la satisfacción del total de los estudiantes comparada con otras variables en este medio (Gunawardena, 2003). El interés en el grado de satisfacción reside en la experiencia de aprendizaje CMC de los estudiantes, caso en el que se han tenido en cuenta las necesidades afectivas en el proceso de aprendizaje, razón por la que estos estudiantes se matricularán en este tipo de experiencias nuevamente y seguirán con cursos posteriores. (Gunawardena, 2003). 
Al profundizar en el tema de la presencia social, es importante incluir los componentes afectivo y de cohesión propuestos por Rourke, Anderson, Garrison \& Archer (2001) y citados por Serrano (2005). Los indicadores afectivos mejoran la participación de los estudiantes, la interactividad entre la comunidad en línea y el componente de cohesión, y producen y establecen lazos entre los miembros del grupo (Serrano, 2005). Tales procesos indican estrategias y mecanismos de motivación, comunicación, participación e interactividad que apuntan al logro educativo.

Rourke et al. (2001) se centraron en la identificación de comportamientos observables utilizados por los estudiantes para proyectarse como personas "reales" e identificaron tres categorías y doce indicadores de la presencia social que a continuación se presentan:

Tabla 1. Categorias y subcategorías

\begin{tabular}{ll} 
Categoría & Indicadores \\
\hline Afectivo & Expresión de emociones \\
& Uso del humor \\
& Auto-revelación \\
& \\
Interactivo & Siguiendo un hilo \\
& Citando a otros mensajes \\
& Hace referencia explícita a otros \\
& mensajes \\
& Hacer una pregunta \\
& Complementando, expresando su \\
& reconocimiento, expresado \\
& \\
Coherentes & Acuerdo \\
Coherentes vocativos \\
Direcciones o se refiere al grupo el uso de \\
pronombres inclusiva \\
Fática / Saludos \\
\\
\\
\\
\\
\end{tabular}

Tu \& McIsaac (2002) más tarde argumentaron el resultado de una mezcla de métodos de estudio que han realizado sobre la presencia social e identificaron las dimensiones y variables de presencia social. 


\section{Martha Liliana Palomino Leiva y Gabriel Mauricio Ramírez Villegas}

La presencia social en ambientes virtuales de aprendizaje: una estrategia de interacción y construcción del conocimiento, artículo producto de la investigación

Tabla 2. Dimensiones y variables de la presencia social

\begin{tabular}{|c|c|c|c|}
\hline I. Contexto social & $\begin{array}{l}\text { II. Comunicación } \\
\text { en línea }\end{array}$ & III. Interactividad & IV. Privacidad \\
\hline $\begin{array}{l}\text { Familiaridad con } \\
\text { beneficiarios }\end{array}$ & $\begin{array}{l}\text { Teclado y habilidades } \\
\text { de precisión }\end{array}$ & Oportuna respuesta & Formatos de CMC \\
\hline Asertivo / aquiescente & $\begin{array}{l}\text { El uso de emoticones y } \\
\text { para lenguaje }\end{array}$ & $\begin{array}{l}\text { Estilos de } \\
\text { comunicación }\end{array}$ & $\begin{array}{l}\text { El acceso y la } \\
\text { ubicación }\end{array}$ \\
\hline $\begin{array}{l}\text { Informal / formal } \\
\text { relación }\end{array}$ & $\begin{array}{l}\text { Características en } \\
\text { tiempo real discusión }\end{array}$ & $\begin{array}{l}\text { Duración de los } \\
\text { mensajes }\end{array}$ & Los patrones de CMC \\
\hline $\begin{array}{l}\text { Relaciones de } \\
\text { confianza }\end{array}$ & $\begin{array}{l}\text { Características de } \\
\text { foros de debate }\end{array}$ & Formal / informal & \\
\hline Relaciones sociales & $\begin{array}{l}\text { Habilidades del } \\
\text { lenguaje } \\
\text { (lectura y escritura) }\end{array}$ & $\begin{array}{l}\text { Tipo de tareas } \\
\text { (planificación, } \\
\text { creatividad, sociales) }\end{array}$ & \\
\hline $\begin{array}{l}\text { Actitud psicológica } \\
\text { hacia la tecnología }\end{array}$ & & Tamaño de los grupos & \\
\hline $\begin{array}{l}\text { El acceso y la } \\
\text { ubicación }\end{array}$ & & $\begin{array}{l}\text { Estrategias de } \\
\text { comunicación }\end{array}$ & \\
\hline $\begin{array}{l}\text { El acceso y la } \\
\text { ubicación }\end{array}$ & & & \\
\hline
\end{tabular}

Serrano (2005), apoyada en la investigación de Rourke et al. (2001), tuvo en cuenta los siguientes indicadores:

Tabla 3. Indicadores de la presencia social

\section{Componente afectivo}

a. Expresiones convencionales o no convencionales de la emoción, incluido el uso repetitivo de puntuación, capitalización visible y emoticones.

b. Uso del humor a través de las burlas, la ironía, la subestimación y el sarcasmo.

c. La auto-revelación de manifiesto en la inclusión de detalles de la vida fuera de la clase o por expresar vulnerabilidad.

\section{Componente de cohesión}

a. Uso de los pronombres integradores de la dirección o la referencia para el grupo. b. Uso de la comunicación fática, o de comunicación que cumple una función puramente social. 
Gándara (1997) define la interacción como la acción recíproca entre dos agentes. En el caso de la interacción en educación a distancia, estos indicadores se constituyen en los más trascendentes en el proceso de aprendizaje, y es además un medio que desarrolla la presencia social.

Respecto a los cursos de investigación cualitativa en línea, se encuentran diversos cursos virtuales que se ofrecen al público pero no se encuentran estudios relacionados con el desarrollo de sus procesos pedagógicos y resultados de aprendizaje en línea. Por ello se tomarán como referencia las investigaciones y resultados de Rourke et al. (2001), Tu \& McIsaac (2002) y Cabrero, Márquez, Miranda \& Espíndola (2008) para desarrollar los procesos de observación de la presencia social con sus dimensiones de afecto, interacción y cohesión de los actores educativos que participan en este curso virtual en la UNAD.

A continuación se presenta la tabla de categorías e indicadores tenidos en cuenta para la observación y el análisis en esta investigación:

Tabla 4. Categorías e Indicadores de la presencia social

\begin{tabular}{|ll}
\hline Categorías & Indicadores \\
\hline Afecto & $\begin{array}{l}\text { Identidad con el grupo } \\
\text { Motivación en los grupos colaborativos } \\
\text { Expresión del humor } \\
\text { Evidencia de frustraciones }\end{array}$ \\
\hline Comunicación & $\begin{array}{l}\text { Tipo de comunicación Frecuencia de } \\
\text { comunicación Calidad de la comunicación }\end{array}$ \\
\hline Interacción & $\begin{array}{l}\text { Tipo de conservaciones establecidas Presencia } \\
\text { de conflictos y abordajes Tipo de participación } \\
\text { Acceso a las estrategias tecno-pedagógicas }\end{array}$ \\
\hline Cohesión & Organización establecida en los grupos \\
& colaborativos \\
Estrategias de trabajo establecidas \\
Cumplimiento de objetivos de aprendizaje
\end{tabular}

\section{Metodología}

La metodología implementada tuvo un abordaje cualitativo y cuantitativo. El primero se dio a partir de la observación y una lista de chequeo elaborada de 
acuerdo con la tabla de categorías e indicadores de presencia social construida por Rourke et al. (2001) con el propósito de evaluar la participación en los foros colaborativos. El cuantitativo se realizó mediante una encuesta con base en la tabla de dimensiones y variables de la presencia social construida por $\mathrm{Tu}$ \& McIsaac (2002) aplicada a los actores educativos del curso virtual.

Las actividades en las que se realizó la recolección de los datos y el análisis respectivo fueron colaborativas como el foro de noticias, el foro general del curso, el foro de reconocimiento de actores, los foros de trabajo colaborativo, la actividad Wiki y el sistema de mensajería interna del curso virtual de investigación cualitativa.

La población objetivo estuvo constituida por los participantes del curso virtual de investigación cualitativa, los estudiantes matriculados en el período 2010I y los tutores asignados a este curso virtual. La interacción se dio entre una directora, dos tutoras y 648 estudiantes. Los últimos se organizaron en 131 grupos de entre tres y cinco estudiantes, como se muestra en la tabla 5. La responsable de todo el curso fue la directora, quien definió estructura, contenidos, actividades y evaluaciones del mismo y además tuvo a cargo 55 grupos para el acompañamiento tutorial. Las tutoras realizaron este acompañamiento $\mathrm{y}$ contribuyeron en los procesos de evaluación del curso.

Tabla 5. Participantes 2010-I

\begin{tabular}{lll} 
Actor educativo & Grupos asignados & $\begin{array}{l}\text { Número de estudiantes } \\
\text { asignados }\end{array}$ \\
\hline Director de curso: Ligia Ortiz & 55 & 268 \\
Tutora: Martha Liliana Palomino & 65 & 325 \\
Tutora: Amparo Dagua & 11 & 55 \\
\hline Total & 131 & 648
\end{tabular}

Fuente: Participantes campus virtual 02 curso de investigación cualitativa 2010 I.

Los estudiantes fueron hombres y mujeres de nacionalidad colombiana dispersos geográficamente, en su mayoría adultos y generalmente, trabajadores que buscan cualificarse y mejorar su nivel de formación académica.

Existe una población de 648 estudiantes y 3 tutores en el curso de Investigación cualitativa. El tamaño de la muestra fue de 241 personas, número al cual se llegó tras aplicar la siguiente fórmula:

$$
n=\frac{z^{2} * p^{*} q * N}{(N-1) * E^{2}+Z^{2} * p * q} \quad n=\frac{1.96^{2} * 0.5 * 0.5 * 651}{(651-1) * 0.05^{2}+1.96^{2} * 0.5 * 0.5}=>n=\frac{625.2204}{2.5854}=>n=241.8273
$$


Para la aplicación de la encuesta se envió a todos los estudiantes seleccionados un mensaje a través del curso virtual, por medio de la mensajería de la plataforma y el correo electrónico, solicitando su diligenciamiento. Para la observación de los foros se tuvieron en cuenta los grupos con mayor interacción según los registros de ingreso y participación del aula y se estableció una lista de chequeo a fin de registrar los indicadores del componente afectivo y de cohesión presentes en los grupos colaborativos seleccionados.

La encuesta se aplicó en línea a través del sitio web e-encuestas, que suministró el siguiente enlace: http://www.e-encuesta.com/answer. do?testid=notoDRGT0Gw=. Este enlace se socializó con los estudiantes y tutores del curso por medio de mensajes de correo electrónico, mensajes en los foros del curso y en la página principal al interior del curso virtual. Permitió la participación de los estudiantes y tutores por un período de veinte días. Posteriormente se recogieron los datos, se tabularon y graficaron. La tabla de verificación se diligenció al observar los grupos seleccionados. Luego se realizó la tabulación sumando los datos recogidos según el indicador de las categorías afectiva, interactiva y cohesiva.

\section{Resultados y discusión:}

Análisis cuantitativo de la percepción de los actores educativos respecto a la presencia social del curso

Para hacer el análisis cuantitativo se aplicó una encuesta a los estudiantes del curso virtual de investigación cualitativa de la Universidad Nacional Abierta y a Distancia, donde se les consultó acerca de la presencia social, la comunicación y la interacción realizada entre los actores educativos que participan en el curso virtual.

Para ello se revisaron las respuestas de 240 estudiantes y dos tutores respecto a las siguientes preguntas:

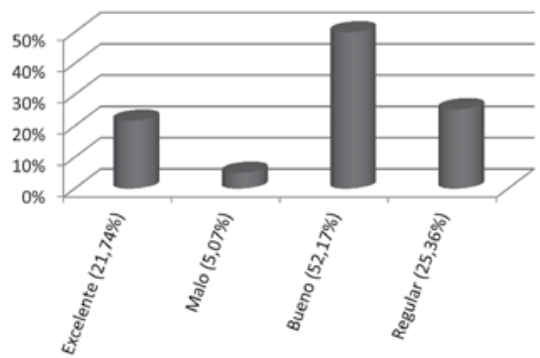

Figura 1. Análisis de la pregunta: ¿Cree usted que la comunicación escrita o verbal de su tutor es? 
La presencia social en ambientes virtuales de aprendizaje: una estrategia de interacción y construcción del conocimiento, artículo producto de la investigación

El 21.74\% de los encuestados cree que la comunicación escrita o verbal con el tutor es excelente. E1 $52.17 \%$ cree que es buena. E1 $25.35 \%$ cree que es regular y sólo el $5.07 \%$ cree que es mala.

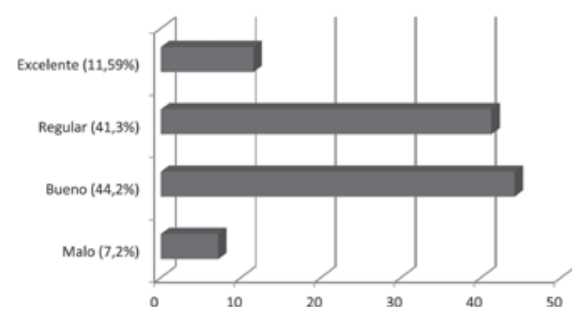

Figura 2. Análisis de la pregunta: ¿Cree usted que la comunicación con sus compañeros de trabajo colaborativo es?

El $11.59 \%$ de los encuestados cree que la comunicación con sus compañeros de grupo de trabajo es excelente. El $44.2 \%$ cree que es buena. El $41.3 \%$ cree que es regular y sólo el $7.25 \%$ cree que es mala.

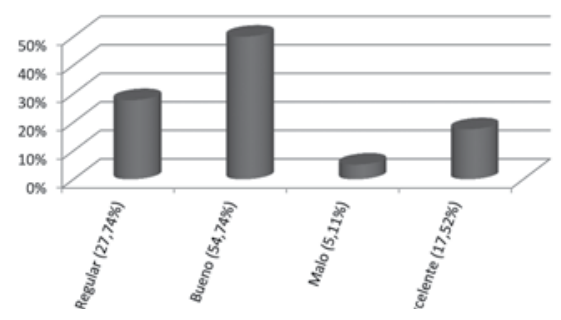

Figura 3. Análisis de la pregunta: ¿Considera que la calidad de la comunicación con su tutor es?

El $17.52 \%$ de los encuestados cree que la calidad de la comunicación con el tutor virtual es excelente. El $54.74 \%$ cree que es buena. El $27.74 \%$ cree que es regular y sólo el $5.11 \%$ cree que es mala.

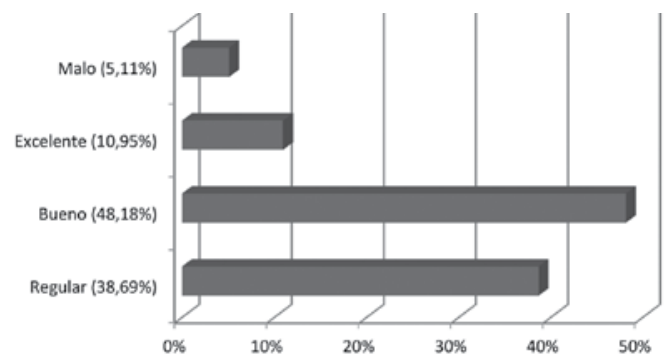

Figura 4. Análisis de la pregunta: ¿Considera que la calidad de la comunicación con sus compañeros de grupo de trabajo colaborativo es? 
El 10.95\% de los encuestados cree que la calidad de la comunicación con sus compañeros de trabajo colaborativo es excelente. El $48.18 \%$ cree que es buena. El $38.69 \%$ cree que es regular y sólo el $5.11 \%$ cree que es mala.

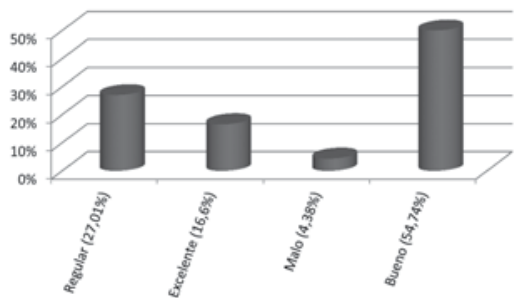

Figura 5. Análisis de la pregunta: ¿Cómo considera la interacción del tutor con los estudiantes del curso virtual?

El $16.06 \%$ de los encuestados cree que la interacción del tutor con los estudiantes es excelente. El $54.74 \%$ cree que es buena. El $27.01 \%$ cree que es regular y sólo el $5.74 \%$ cree que es mala.

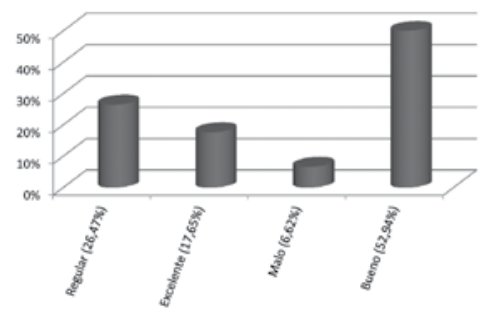

Figura 6. Análisis de la pregunta: ¿Cómo es la interacción con los compañeros de grupo de trabajo colaborativo?

El 11.68\% de los encuestados cree que la interacción con los compañeros de trabajo colaborativo es excelente. El $43.61 \%$ cree que es buena. El $41.61 \%$ cree que es regular y sólo el $6.57 \%$ cree que es mala.

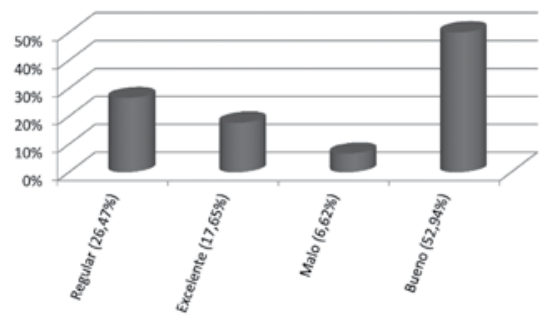

Figura 7. Análisis de la pregunta: ¿Considera usted que el tiempo de respuesta es? 
La presencia social en ambientes virtuales de aprendizaje: una estrategia de interacción y construcción del conocimiento, artículo producto de la investigación

El $17.65 \%$ de los encuestados cree que el tiempo de respuesta del tutor es excelente. El $52.94 \%$ cree que es bueno. El $26.47 \%$ cree que es regular y sólo el $6.62 \%$ cree que es malo.

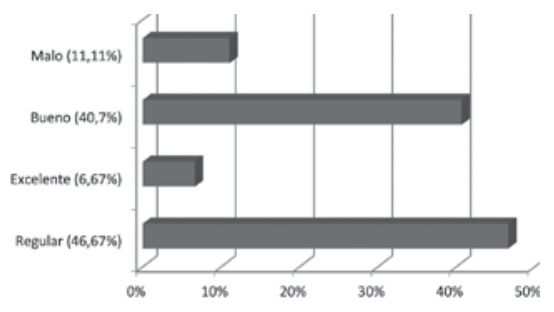

Figura 8. Análisis de la pregunta: ¿Considera usted que el tiempo de respuesta de sus compañeros en el desarrollo de las actividades colaborativas es?

El $6.67 \%$ de los encuestados cree que el tiempo de respuesta de sus compañeros en el desarrollo del trabajo colaborativo es excelente. El $40.09 \%$ cree que es bueno. El $46.67 \%$ cree que es regular y sólo el $11.11 \%$ cree que es malo.

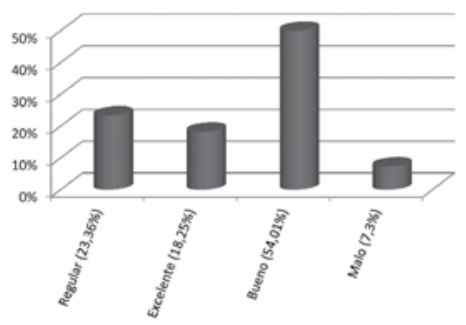

Figura 9. Análisis de la pregunta: ¿Considera usted que las actividades desarrolladas utilizando los foros dentro del curso virtual son?

El $18.25 \%$ de los encuestados cree que las actividades desarrolladas utilizando los foros del curso virtual son excelentes. El 54.01\% cree que son buenas. El $28.25 \%$ cree que son regulares y sólo el $7.3 \%$ cree que son malas.

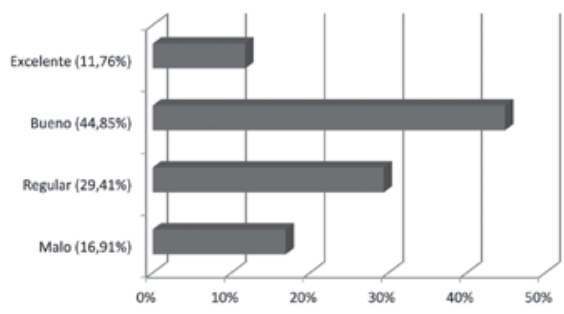

Figura 10. Análisis de la pregunta: ¿Considera que la actividad Wiki del curso virtual le ayuda en el desarrollo de las actividades colaborativas de forma? 
El $11.76 \%$ de los encuestados cree que la actividad wiki le ayuda en el desarrollo de las actividades de forma excelente. El $44.85 \%$ cree que de forma buena. El $29.41 \%$ cree que regular y sólo el $16.91 \%$ cree que mala.

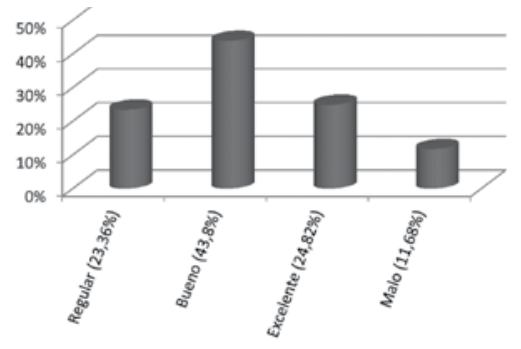

Figura 11. Análisis de la pregunta: ¿Considera que el sistema de mensajería del curso virtual es?

El $24.82 \%$ de los encuestados cree que el sistema de mensajería del curso virtual es excelente. El $43.8 \%$ cree que es bueno. El $23.36 \%$ cree que es regular y sólo el $11.68 \%$ cree que es malo.

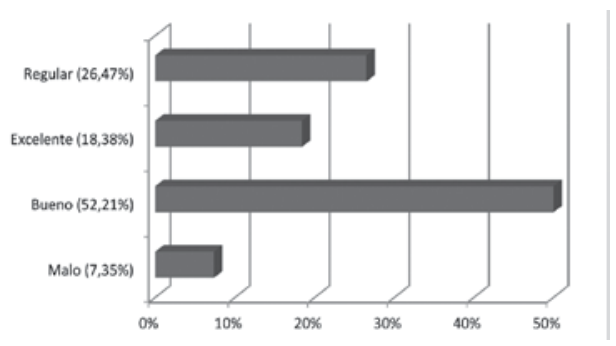

Figura 12. Análisis de la pregunta: ¿Considera usted que la relación que se produce con su tutor de curso virtual es?

El $18.38 \%$ de los encuestados cree que la relación con su tutor virtual es excelente. El $52.21 \%$ cree que es buena. El $26.47 \%$ cree que es regular y sólo el $7.35 \%$ cree que es mala.

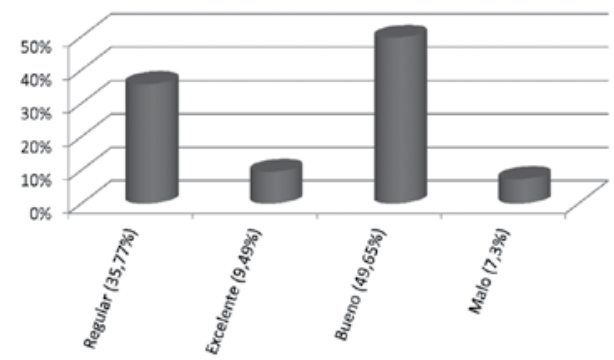

Figura 13. Análisis de la pregunta:¿Considera usted que la relación que se genera con sus compañeros de grupo colaborativo es? 
El $9.49 \%$ de los encuestados cree que la relación que se genera con sus compañeros de grupo colaborativo es excelente. El $49.64 \%$ cree que es buena. El $35.77 \%$ cree que es regular y sólo el $7.3 \%$ cree que es mala.

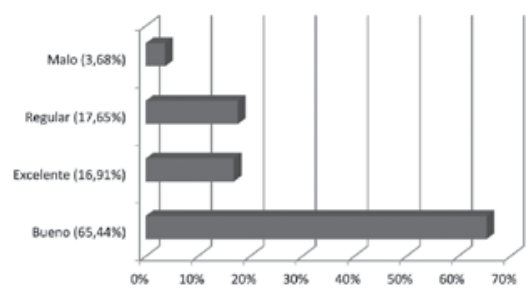

Figura 14. Análisis de la pregunta: ¿Considera usted que los productos finales enviados por su grupo de trabajo colaborativo son?

El $16.91 \%$ de los encuestados considera que los productos finales enviados por su grupo de trabajo colaborativo son excelentes. El $65.44 \%$ cree que son buenos. El $17.65 \%$ cree que son regulares y sólo el 3.6\% cree que son malos.

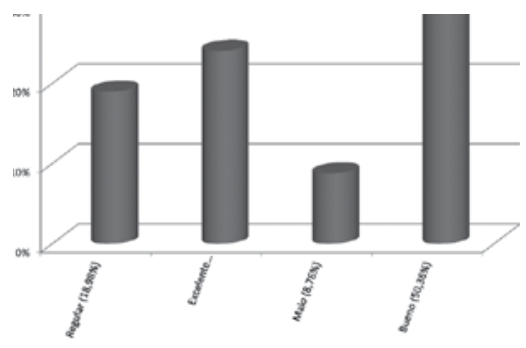

Figura 15. Análisis de la pregunta: ¿Considera usted que el aprendizaje adquirido en el curso virtual a través del desarrollo del trabajo colaborativo es?

El $24.09 \%$ de los encuestados considera que el aprendizaje adquirido en el curso virtual a través del desarrollo del trabajo colaborativo es excelente. El $50.36 \%$ cree que es bueno. El $18.98 \%$ cree que es regular y sólo el $8.76 \%$ cree que es malo.

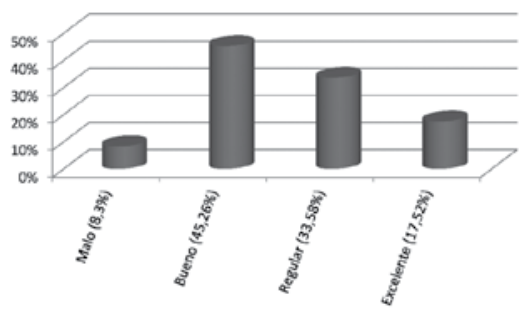

Figura 16. Análisis de la pregunta: ¿Considera usted que la realimentación de los trabajos colaborativos de su tutor hacia el trabajo del grupo es? 
El 17.52\% de los encuestados considera que la realimentación de los trabajos colaborativos de su tutor es excelente. El $45.26 \%$ cree que es buena. El 33.58\% cree que es regular y sólo el $8.03 \%$ cree que es mala.

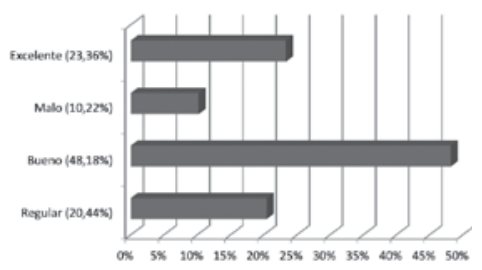

Figura 17. Análisis de la pregunta: ¿Se siente satisfecho con el aprendizaje adquirido en el desarrollo del curso virtual?

El $23.36 \%$ de los encuestados siente una satisfacción excelente con el aprendizaje adquirido en el desarrollo del curso virtual. El 48.18\% siente que es buena. El 20.44\% siente que es regular y sólo el $10.22 \%$ siente que es mala.

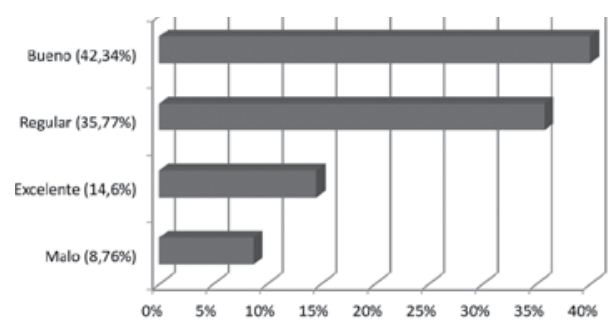

Figura 18. Análisis de la pregunta: ¿Considera que ha generado comunidades de aprendizaje con los compañeros de grupo y con el tutor del curso virtual de manera?

El $14.6 \%$ de los encuestados considera que ha generado comunidades de aprendizaje con los compañeros de grupo y con el tutor virtual de forma excelente. El $42.34 \%$ lo considera bueno. El $35.77 \%$ lo considera regular y sólo el $8.76 \%$ lo considera mala.

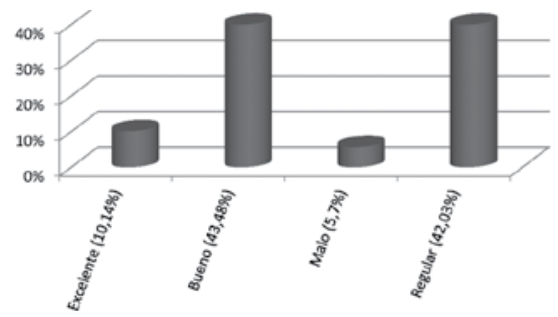

Figura 19. Análisis de la pregunta: ¿Considera que las discusiones realizadas en el grupo colaborativo son? 
El $10.14 \%$ de los encuestados considera que las discusiones realizadas en el grupo colaborativo son excelentes. El $43.48 \%$ considera las discusiones buenas. El $42.03 \%$ considera las discusiones regulares y sólo el $5.07 \%$ considera las discusiones malas.

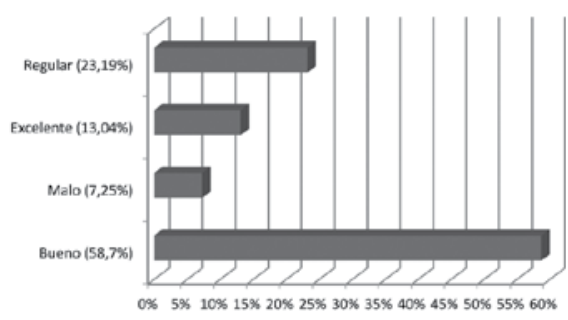

Figura 20. Análisis de la pregunta: ¿Considera que los comentarios del tutor sobre sus tareas son?

El 13.04\% de los encuestados considera que los comentarios del tutor sobre sus tareas son excelentes. El 58.7\% considera los comentarios buenos. El $23.19 \%$ considera los comentarios regulares y sólo el $7.25 \%$ considera los comentarios malos.

Análisis cualitativo de la presencia social de los actores educativos en el desarrollo de los foros colaborativos

Para realizar este análisis se observó el foro general del curso, el foro de reconocimiento general y de actores y los foros colaborativos 1 y 2 , lo que permitió la descripción de las categorías de presencia social (afecto, interacción y cohesión) definidas por García, Márquez, Miranda \& Espíndola (2008). Esta descripción se realizó a partir de la verificación de indicadores de cada una de las categorías dadas a través de una lista de chequeo como se muestra en las tablas 1 y 2 .

La tabla 6 presenta las categorías e indicadores de la presencia social observados a partir de la interacción desarrollada por los estudiantes, la directora del curso y las dos tutoras en el foro general del curso.

Tabla 6. Presencia social de los participantes en el foro general del curso

\begin{tabular}{|l|l|l|l|l|}
\hline \multicolumn{1}{|c|}{ CATEGORÍAS E INDICADORES } & SI & NO & NA & $\begin{array}{c}\text { OBSERVACIONES Y } \\
\text { COMENTARIOS }\end{array}$ \\
\hline AFECTO & & & & \\
\hline $\begin{array}{l}\text { Se identifican claramente los miembros } \\
\text { del grupo }\end{array}$ & 6 & 1 & 1 & \\
\hline
\end{tabular}




\begin{tabular}{|c|c|c|c|c|}
\hline CATEGORÍAS E INDICADORES & SI & NO & NA & $\begin{array}{l}\text { OBSERVACIONES Y } \\
\text { COMENTARIOS }\end{array}$ \\
\hline $\begin{array}{l}\text { Se actualizan los perfiles de cada } \\
\text { miembro }\end{array}$ & 7 & 0 & 2 & \\
\hline $\begin{array}{l}\text { Se evidencia identidad o pertinencia con } \\
\text { el grupo }\end{array}$ & 6 & 1 & 2 & \\
\hline Se usan emoticones & 3 & 6 & 0 & \\
\hline Se expresa sentido del humor & 0 & 9 & 0 & \\
\hline $\begin{array}{l}\text { Se revelan frustraciones entre los miem- } \\
\text { bros de grupo }\end{array}$ & 7 & 2 & 0 & \\
\hline $\begin{array}{l}\text { Se revelan temas o conversaciones de la } \\
\text { vida cotidiana }\end{array}$ & 1 & 8 & 0 & \\
\hline $\begin{array}{l}\text { Se evidencia actitud positiva frente al } \\
\text { curso }\end{array}$ & 8 & 0 & 1 & \\
\hline \multicolumn{5}{|l|}{ INTERACCION } \\
\hline $\begin{array}{l}\text { Se establecen conversaciones } \\
\text { únicamente en el tema del foro. }\end{array}$ & 4 & 5 & 0 & \\
\hline $\begin{array}{l}\text { Se establece conversaciones sincrónica } \\
\text { o asincrónica fuera del aula virtual. (Blog, } \\
\text { wikis, redes sociales, correo electrónico, } \\
\text { audio foros, etc.) }\end{array}$ & 4 & 5 & & $\begin{array}{l}\text { Se establece en audio foro en } \\
\text { woxopop pero no se desarrolla. } \\
\text { Se usa intensamente el correo } \\
\text { electrónico interno del curso }\end{array}$ \\
\hline $\begin{array}{l}\text { Se establecen conversaciones diferentes } \\
\text { a las académicas }\end{array}$ & 1 & 8 & 0 & $\begin{array}{l}\text { Se habilita un tema sobre Día de } \\
\text { la mujer. }\end{array}$ \\
\hline $\begin{array}{l}\text { Se resuelven adecuadamente los } \\
\text { conflictos entre los compañeros }\end{array}$ & 3 & 1 & 5 & \\
\hline $\begin{array}{l}\text { Se definen estrategias de comunicación } \\
\text { entre los miembros. }\end{array}$ & 3 & 2 & 6 & \\
\hline $\begin{array}{l}\text { Se mantiene el debate entre los } \\
\text { miembros del grupo. }\end{array}$ & 2 & 4 & 3 & \\
\hline $\begin{array}{l}\text { Se evidencia participación activa de } \\
\text { todos los miembros del grupo }\end{array}$ & 4 & 5 & 0 & \\
\hline $\begin{array}{l}\text { Se evidencia dificultades en el manejo } \\
\text { tecnológico o ingreso al curso }\end{array}$ & 8 & 1 & 0 & \\
\hline COHESION & & & & \\
\hline
\end{tabular}


La presencia social en ambientes virtuales de aprendizaje: una estrategia de interacción y construcción del conocimiento, artículo producto de la investigación

\begin{tabular}{|l|l|l|l|l|}
\hline CATEGORÍAS E INDICADORES & SI & NO & NA & $\begin{array}{l}\text { OBSERVACIONES Y } \\
\text { COMENTARIOS }\end{array}$ \\
\hline $\begin{array}{l}\text { Se definen claramente los objetivos del } \\
\text { foro }\end{array}$ & 6 & 2 & 1 & Por lo general el director o tutor \\
\hline $\begin{array}{l}\text { Se asignan roles de trabajo entre los } \\
\text { participantes }\end{array}$ & 0 & 6 & 3 & \\
\hline $\begin{array}{l}\text { Se generan responsabilidades entre los } \\
\text { participantes para el desarrollo de las } \\
\text { tareas o actividades }\end{array}$ & 0 & 5 & 4 & \\
\hline $\begin{array}{l}\text { Se definen claramente estrategias de } \\
\text { trabajo }\end{array}$ & 0 & 5 & 4 & \\
\hline $\begin{array}{l}\text { Se hace realimentación a los aportes } \\
\text { consignados por parte de los compañe- } \\
\text { ros de grupo. }\end{array}$ & 1 & 7 & 1 & \\
\hline $\begin{array}{l}\text { Se hace realimentación a los aportes } \\
\text { consignados por parte del tutor }\end{array}$ & 9 & 0 & 0 & \\
\hline \begin{tabular}{l} 
Se logran los objetivos del foro \\
\hline
\end{tabular} & 6 & 3 & 0 & \\
\hline
\end{tabular}

Tabla 7. Presencia social de los participantes en los foros colaborativos

\begin{tabular}{|l|l|l|l|l|}
\hline CATEGORÍAS E INDICADORES & SI & NO & NA & $\begin{array}{l}\text { OBSERVACIONES Y } \\
\text { COMENTARIOS }\end{array}$ \\
\hline AFECTO & & & & \\
\hline $\begin{array}{l}\text { Se identifican claramente los miembros } \\
\text { del grupo }\end{array}$ & 39 & 1 & 0 & $\begin{array}{l}\text { En algunos grupos solo participan } \\
\text { algunos miembros. }\end{array}$ \\
\hline $\begin{array}{l}\text { Se actualizan los perfiles de cada } \\
\text { miembro }\end{array}$ & 30 & 10 & 0 & Solo algunos actualizan el perfil. \\
\hline $\begin{array}{l}\text { Se evidencia identidad o pertinencia con } \\
\text { el grupo }\end{array}$ & 36 & 4 & 0 & $\begin{array}{l}\text { Se hace al final por el ingreso tarde de } \\
\text { los compañeros de grupo }\end{array}$ \\
\hline Se usan emoticones & 7 & 33 & 0 & $\begin{array}{l}\text { Solo uno o dos estudiantes. Usan la } \\
\text { carita feliz. En un grupo no utilizan } \\
\text { emoticones sino frases celebres por } \\
\text { cada participante. Por lo general } \\
\text { un miembro del grupo motiva a los } \\
\text { compañeros a participar del foro. }\end{array}$ \\
\hline
\end{tabular}




\begin{tabular}{|c|c|c|c|c|}
\hline CATEGORÍAS E INDICADORES & SI & NO & NA & $\begin{array}{l}\text { OBSERVACIONES Y } \\
\text { COMENTARIOS }\end{array}$ \\
\hline Se expresa sentido del humor & 5 & 35 & 0 & \\
\hline $\begin{array}{l}\text { Se revelan frustraciones entre los miem- } \\
\text { bros de grupo }\end{array}$ & 16 & 24 & 0 & $\begin{array}{l}\text { Se revelan por el ingreso tarde al } \\
\text { foro y algunos por el no dominio del } \\
\text { programa Cmaptools. }\end{array}$ \\
\hline $\begin{array}{l}\text { Se revelan temas o conversaciones de la } \\
\text { vida cotidiana }\end{array}$ & 6 & 34 & 0 & \\
\hline $\begin{array}{l}\text { Se evidencia actitud positiva frente al } \\
\text { curso }\end{array}$ & 36 & 4 & 0 & \\
\hline \multicolumn{5}{|l|}{ INTERACCION } \\
\hline $\begin{array}{l}\text { Se establecen conversaciones única- } \\
\text { mente en el tema del foro. }\end{array}$ & 35 & 3 & 0 & $\begin{array}{l}\text { Un foro no se habilitó por la tutora. Un } \\
\text { grupo no inicio realizó debate ni en el } \\
\text { foro de reconocimiento ni en el trabajo } \\
\text { colaborativo } 1 .\end{array}$ \\
\hline $\begin{array}{l}\text { Se establece conversaciones sincrónica } \\
\text { o asincrónica fuera del aula virtual. (Blog, } \\
\text { wikis, redes sociales, correo electrónico, } \\
\text { audio foros, etc.) }\end{array}$ & 11 & 29 & 0 & $\begin{array}{l}\text { Posiblemente porque se socializan } \\
\text { correos electrónicos y No. telefónico } \\
\text { o celular. Algunos comparten pagina } \\
\text { web personal o institucional. }\end{array}$ \\
\hline $\begin{array}{l}\text { Se establecen conversaciones diferentes } \\
\text { a las académicas }\end{array}$ & 8 & 32 & 0 & \\
\hline $\begin{array}{l}\text { Se resuelven adecuadamente los con- } \\
\text { flictos entre los compañeros }\end{array}$ & 13 & 11 & 16 & $\begin{array}{l}\text { No se evidencian. Cuando se pre- } \\
\text { sentan es por el ingreso tardío de los } \\
\text { compañeros al foro. }\end{array}$ \\
\hline $\begin{array}{l}\text { Se definen estrategias de comunicación } \\
\text { entre los miembros. }\end{array}$ & 17 & 23 & 0 & $\begin{array}{l}\text { Algunas veces se indica fecha y hora } \\
\text { de ingreso }\end{array}$ \\
\hline $\begin{array}{l}\text { Se mantiene el debate entre los miem- } \\
\text { bros del grupo. }\end{array}$ & 18 & 22 & 0 & $\begin{array}{l}\text { Se mantiene conversación sin debate } \\
\text { a los aportes. Se realiza un poco } \\
\text { tarde. Se colocan los aportes indivi- } \\
\text { duales y se responde en cuanto a lo } \\
\text { metodológico pero no se debate el } \\
\text { contenido. Muchos aportes individua- } \\
\text { les sin discusión }\end{array}$ \\
\hline $\begin{array}{l}\text { Se evidencia participación activa de } \\
\text { todos los miembros del grupo }\end{array}$ & 18 & 22 & 0 & $\begin{array}{l}\text { La mayoría la realiza casi al cierre del } \\
\text { foro. En algunos grupos solo algunos } \\
\text { miembros. }\end{array}$ \\
\hline
\end{tabular}




\section{Martha Liliana Palomino Leiva y Gabriel Mauricio Ramírez Villegas}

La presencia social en ambientes virtuales de aprendizaje: una estrategia de interacción y construcción del conocimiento, artículo producto de la investigación

\begin{tabular}{|c|c|c|c|c|}
\hline CATEGORÍAS E INDICADORES & SI & NO & NA & $\begin{array}{l}\text { OBSERVACIONES Y } \\
\text { COMENTARIOS }\end{array}$ \\
\hline $\begin{array}{l}\text { Se evidencia dificultades en el manejo } \\
\text { tecnológico o ingreso al curso }\end{array}$ & 36 & 4 & 0 & $\begin{array}{l}\text { Uso del programa Cmaptools. Pro- } \\
\text { blemas de conectividad. Campus no } \\
\text { disponible y resultados de lecciones o } \\
\text { quices con } 0 \text { de calificación }\end{array}$ \\
\hline \multicolumn{5}{|l|}{ COHESION } \\
\hline $\begin{array}{l}\text { Se definen claramente los objetivos del } \\
\text { foro }\end{array}$ & 34 & 6 & 0 & $\begin{array}{l}\text { Son definidos por la directora y la tu- } \\
\text { tora. Un grupo el foro no fue habilitado } \\
\text { por la tutora. }\end{array}$ \\
\hline $\begin{array}{l}\text { Se asignan roles de trabajo entre los } \\
\text { participantes }\end{array}$ & 15 & 25 & 0 & $\begin{array}{l}\text { Sobre todo el líder y el compilador del } \\
\text { trabajo final. Solo algunos miembros } \\
\text { lo definen por voluntad propia y otros } \\
\text { designan. Poco se acuerdan estos } \\
\text { roles. }\end{array}$ \\
\hline $\begin{array}{l}\text { Se generan responsabilidades entre los } \\
\text { participantes para el desarrollo de las } \\
\text { tareas o actividades }\end{array}$ & 15 & 25 & 0 & $\begin{array}{l}\text { Solo algunos miembros lo definen } \\
\text { por voluntad propia y otros designan. } \\
\text { Poco se acuerdan las responsabili- } \\
\text { dades. }\end{array}$ \\
\hline $\begin{array}{l}\text { Se definen claramente estrategias de } \\
\text { trabajo }\end{array}$ & 11 & 29 & 0 & $\begin{array}{l}\text { Se definen en el transcurso de las } \\
\text { participaciones en el foro. }\end{array}$ \\
\hline $\begin{array}{l}\text { Se hace realimentación a los aportes } \\
\text { consignados por parte de los compañe- } \\
\text { ros de grupo. }\end{array}$ & 17 & 23 & 0 & $\begin{array}{l}\text { Solo algunos estudiantes. Pocas } \\
\text { veces sobre el contenido. Se hace } \\
\text { llamado a los compañeros para } \\
\text { que realicen su participación y se } \\
\text { responde más por el no ingreso al } \\
\text { foro. Se comunican con el tutor con } \\
\text { gratitud por la ayuda brindada. }\end{array}$ \\
\hline $\begin{array}{l}\text { Se hace realimentación a los aportes } \\
\text { consignados por parte del tutor }\end{array}$ & 8 & 32 & 0 & $\begin{array}{l}\text { Solo al inicio del foro. Muy pocas } \\
\text { veces en el desarrollo del foro. }\end{array}$ \\
\hline Se logran los objetivos del foro & 31 & 9 & 0 & $\begin{array}{l}\text { El foro de reconocimiento la mayoría } \\
\text { realiza su aporte individual. Solo } \\
\text { algunos grupos consolidan el final ya } \\
\text { sea por la vocería o el liderazgo de } \\
\text { uno de los miembros. No se define } \\
\text { trabajo grupal propiamente dicho. } \\
\text { No se evidencia un trabajo grupal } \\
\text { organizado. }\end{array}$ \\
\hline
\end{tabular}


En este foro se encontraron nueve (9) temas habilitados, tres (3) de presentación, uno (1) sobre problemas para activación de actividades, uno (1) sobre ampliación de fechas de trabajos colaborativos, uno (1) sobre herramientas para elaborar la tarea del reconocimiento, uno (1) sobre el día de la mujer y un (1) audio foro. En estos foros la interacción se evidencia más en los temas de presentación, en los problemas para activación de actividades y en la ampliación de fechas de trabajos colaborativos dado que hubo una participación aproximada de 188 estudiantes. En este foro se evidencia una gran presencia tanto de la directora como de las tutoras del curso.

La tabla 7 presenta las categorías e indicadores de la presencia social observada en los tres foros colaborativos desarrollados actualmente y orientados por una de las tutoras del curso (reconocimiento general de actores, trabajo colaborativo 1 y trabajo colaborativo 2). En estos foros se encontraron uno o dos temas habilitados respectivamente. Se observaron cuarenta (40) grupos escogidos al azar de 66 registrados en el aula.

De acuerdo con los datos presentados en la tabla 1 y la tabla 2 , se evidencia un alto desarrollo de la categoría afectiva e interactiva y bajo de la categoría cohesiva. En la afectiva se destaca un alto cumplimiento de los indicadores de identidad personal dada por la actualización del perfil, pertinencia y actitud positiva frente al curso y al grupo colaborativo pero bajo en el uso de emoticones, la expresión del humor y conversaciones de la vida cotidiana. Sólo en siete (7) grupos, un (1) estudiante utiliza la carita feliz como expresión de alegría y gratitud como se indica a continuación:

Hola soy XX, tengo 20 años soy muy alegre, creativa, me encanta escuchar música y leer, me gusta relacionarme con las personas. Espero seamos buenos compañeros los invito a conocerme un poco, mas espero revisen mi trabajo yo revisé el de ustedes, eso me ayuda a conocer su forma de ser. Gusto en conocerlos.

Se encontró que un grupo no usa emoticones sino frases célebres de motivación como estas:

Los amigos sólo se tienen una sola vez en la vida; la libertad nace, crece, vive y muere en el alma; el amor es el coleccionista de una ilusión que no tiene fin", entre otras.

Entre las frustraciones que más se revelan se encuentran el registro de cero en calificaciones o la no realización de lecciones evaluativas y "quizzes" debido al cierre de estos. También se puede deber al no ingreso o a la participación inoportuna de los compañeros del grupo colaborativo. Entre ellas se destacan los siguientes fragmentos: 
... muy buenas tardes, por motivos ajenos a mi voluntad, más específicamente por daños en la red, no pude conectarme los días anteriores, hoy se me da la oportunidad de entrar y doy con la sorpresa que las lecciones y el Quiz de la unidad 2 ya han sido cerradas. Será que hay alguna posibilidad que sean habilitadas nuevamente para poderlas presentar. Muchas gracias.

Profe: tengo un problema, ayer miércoles 17 de marzo conteste el Quiz 1 pero no me lo califico, no entiendo qué pasó, me puede ayudar con eso por favor o lo tengo que volver a contestar?

En la categoría interactiva se evidencia una participación activa sobre todo en los temas de presentación, en los problemas de activación de "quizzes", lecciones evaluativas y ampliación de las actividades del curso, aunque no se muestra una alta participación de todos los estudiantes registrados en el curso. Por lo general ingresan 2, 3 ó 4 de los 5 inscritos en el grupo. Se establecen conversaciones asincrónicas a partir del correo interno del curso o del correo personal entre estudiante-estudiante y estudiante-tutor, con relación a las frustraciones, solicitudes o requerimientos del curso. Muy pocos estudiantes socializan página web personal o institucional, Messenger o Skype. Ningún grupo hace uso de la wiki del curso.

En el audio foro y en las herramientas para elaborar la tarea del foro general del curso no se encuentran respuestas o solicitudes al respecto. Esto muestra varias conversaciones con relación a las dificultades presentadas pero pocas sobre los contenidos y estrategias de aprendizaje del curso. Poco se sostiene una conversación o discusión académica. En los foros colaborativos algunos miembros sostienen una participación activa mientras que otros sólo una vez.

Se indica un alto índice de dificultades tecnológicas o de ingreso al curso debido a la baja conectividad o al no registro de sus resultados académicos de lecciones evaluativas y "quizzes" o a la no revisión de contenidos o guías de aprendizaje. Entre ellas se destacan los siguientes fragmentos:

Profe, estoy bajando la guía de actividades y se me presenta un error al tratar de visualizarla para imprimirla, por favor me puede colaborar para empezar a estudiar, ya que estoy un poco atrasada.

Dra. Buenas noches, realizo la misma petición de mis compañeros, agradezco por favor nos abran nuevamente las actividades de la segunda unidad, el reconocimiento, el Quiz, ya que por problemas de la página ha sido imposible. ... las abrieron el sábado, pero fue imposible realizarlas.

Se definen algunas estrategias de comunicación alternas al foro como el uso del teléfono o correo electrónico personal. Por lo general un estudiante toma 
la vocería, invita y motiva la participación pero cuando no se hace a tiempo se genera conflicto entre los miembros del grupo. En el cumplimiento de estos foros, sobre todo en el foro general de reconocimiento y el trabajo colaborativo 1, la mayoría de los estudiantes presenta su aporte individual, ya casi a la hora del cierre sin una realimentación al respecto. Por lo general estos foros colaborativos de aprendizaje se realizan sin la presencia del tutor.

La categoría cohesiva exhibe un bajo nivel al no mostrarse un alto desarrollo de sus indicadores. Entre los que más se destacan se encuentra la definición de los objetivos del foro y de los temas habilitados, definidos por el tutor como su realimentación frente a las inquietudes de los estudiantes en el foro general o por medio o del correo interno del curso. En el foro general es importante mencionar que los estudiantes no hacen uso adecuado de los temas y plantean sus inquietudes sin atender a la pertenencia o a un objetivo.

En los foros colaborativos no se establecen claramente los roles ni las estrategias de trabajo. Por lo general un estudiante asume el rol de líder o compilador de manera voluntaria, mientras que en otros grupos cada estudiante hace su aporte individual sin que se genere realimentación o discusión grupal, y al final consolidan la información. La tutora hace realimentación a los grupos que solicitan su orientación o aprobación de sus aportes pero esporádicamente. Entre los fragmentos alusivos a lo anterior, se presenta éste:

En la actividad anterior -de reconocimiento del curso-, asumí el rol de líder de grupo, en esta ocasión me postulo para encargarme de la recopilación, organización y si lo desean de entrega del trabajo final. Dejo a su consideración esta propuesta, acompañada de los mejores deseos para tod@s (sic).

\section{Conclusiones}

Los resultados destacan la importancia de las estrategias afectivas e interactivas para el desarrollo de la presencia social en el curso. Entre las estrategias afectivas se destacan la identidad personal, los saludos de bienvenida y de motivación entre los miembros de los grupos colaborativos y el tutor. Estas contribuyen a establecer lazos de amistad y de cooperación entre los mismos pero no favorecen intimidad y cercanía entre los participantes.

Existen deficiencias en los procesos de comunicación entre los actores educativos tutor(a) estudiante, estudiante-estudiante. La relación tutor(a)estudiante se establece a partir de las dificultades presentadas en el curso pero no se desarrolla debate en torno a las temáticas de estudio. Los estudiantes en 
general consideran buena la comunicación en el curso pero no se llega a un nivel de excelencia.

Siguiendo los planteamientos de Gunawardena et al. (1997), Järvelä \& Häkkinen (2002), Garrison et al. (2000) y Lemke (1997) en cuanto al estilo y calidad de la comunicación de estudiantes y tutores, es interesante analizar el contenido de los mensajes, las argumentaciones y estrategias discusivas que se utilizan. Muchos posibilitan el acercamiento entre los miembros mientras que otros los distancian, lo que apoya poco la construcción de los productos y el logro del aprendizaje colaborativo.

Entre las estrategias de comunicación más usadas por los estudiantes al interior del curso se destacan el correo electrónico interno del curso, el correo personal y el teléfono móvil. De hecho, los estudiantes consideran entre bueno y excelente el sistema de mensajería del curso virtual. Los tutores usan con frecuencia el foro general del curso, buscando dar respuesta a las solicitudes de los estudiantes y promoviendo el debate a nivel general, aunque muy pocos estudiantes participan en él. De ahí que sea necesario reorientar los temas de debate al igual que las estrategias discursivas y tecnológicas, las cuales deben ser más incluyentes, reales e ilustrativas.

La presencia del tutor en los foros colaborativos es inusual, aunque un gran porcentaje lo califica como bueno. Se da muchas veces por solicitud de los estudiantes cuando se busca resolver un conflicto grupal o la realimentación de los avances de aprendizaje. El tiempo de respuesta de los compañeros del grupo colaborativo es considerado como regular y malo. Esto hace que se requiera desarrollar habilidades sociales que permitan mejorar el clima social entre los miembros de grupo.

La presencia del tutor en los foros colaborativos puede mejorar la comunicación entre los estudiantes, promover su activa participación y disminuir los conflictos grupales en los foros colaborativos. Éstos ocurren porque los grupos son establecidos por un agente externo, en este caso el director de grupo, sin que se conozcan las afinidades y los acercamientos previos entre sí. Los miembros presentan diversas personalidades, actitudes, percepciones y disposiciones. Ello exige una respuesta recíproca para ayudar a desarrollar mecanismos de autoconocimiento, autorreflexión y autosuperación.

Los grupos colaborativos tienen poca cohesión a la hora de desarrollar el aprendizaje colaborativo. Es más notorio el avance en mentes aisladas y pensamientos individuales, lo que hace que los objetivos de los foros colaborativos no se logren a cabalidad. Uno de los factores es el bajo nivel de comunicación asincrónica entre los miembros de grupo. La mayoría de los estudiantes ingresa casi al final del cierre con sus aportes pero poco se discuten sus contenidos. 
En cuanto a los procesos de interacción, tanto en el desarrollo del curso como en los grupos colaborativos, es importante tener en cuenta los conocimientos técnicos de su manejo y el acceso a la conectividad. Tal vez están incidiendo no sólo en el ingreso al curso sino en la actitud, la motivación y la participación del estudiante. En el logro del aprendizaje colaborativo, la interacción cumple un papel fundamental pero en este caso se requiere mayor motivación y que haya un hilo conductor.

De hecho, Lowe \& Anderson (1997) — citados por García, Márquez, Bustos, Miranda \& Espíndola (2008) - afirman que sólo en el contexto de diseños instruccionales que hacen un uso efectivo de los recursos tecnológicos, y donde los profesores crean un ambiente que conduce a una alta interactividad y autonomía por parte del aprendiz, la interactividad constituye un factor real en la construcción de conocimientos.

Los estudiantes consideran buenas y excelentes las actividades colaborativas desarrolladas en los foros del curso virtual, lo que indica interés de los estudiantes para desarrollarlas pero no se citan los mensajes anteriormente leídos, ni se complementan, ni se cuestionan, ni se referencian. En este aspecto se debe resaltar la importancia de la administración de la agenda para asegurar participaciones a tiempo que permitan alcanzar el logro del aprendizaje, entendiendo que la interacción es un componente fundamental en el desarrollo del pensamiento crítico y reflexivo.

Por esta razón se requiere implementar estrategias que permitan definir roles de trabajo, estrategias de debate, tutoriales de ingreso al aula y uso de los recursos tecnológicos, procesos de inducción adecuados y actitudes de responsabilidad de los miembros participantes. Para ello se requiere mejorar las relaciones de confianza y las relaciones sociales que posibiliten un acercamiento adecuado y el reconocimiento del otro como sujeto que merece respeto y apoyo.

La estrategia de trabajo colaborativo que más se utiliza en el curso son los foros colaborativos habilitados por el tutor. La wiki es otro recurso considerado como bueno por la mayoría de los estudiantes pero muy pocos grupos lo usan. No se utiliza el blog ni el audio foro como estrategias permanentes de aprendizaje aunque sí los emplean algunos estudiantes y tutores en el aula.

Es fundamental analizar cómo el diseño instruccional incide en la motivación, la comunicación y la interacción. Chickering \& Ehrmann. (1996), citado por García, Márquez, Bustos, Miranda \& Espíndola (2008), manifiestan que si el diseño instruccional se ajusta particularmente bien a las necesidades de los alumnos, es posible promover el aprendizaje, especialmente debido a la naturaleza asíncrona de la comunicación, la cual favorece el logro de niveles más altos de reflexión sobre los contenidos del curso. 
En cuanto a la generación de comunidades de aprendizaje en los grupos colaborativos los factores interacción, comunicación y participación en el curso están contribuyendo poco en su consolidación. De hecho, se requiere fortalecer la presencia social de los actores educativos a través de intercambios sociales y relaciones positivas que permitan crear un ambiente de auténtica comunidad.

La presencia social incide notablemente en el desarrollo de las actividades colaborativas del curso. Sobresalen la identidad personal, la motivación individual y grupal, los procesos de comunicación y la participación. Por ello es necesario mejorar el tiempo de respuesta de los foros colaborativos, promover un discurso crítico y establecer procesos de interacción y de participación recíproca a fin de establecer verdaderas comunidades de aprendizajes.

Entre los factores que se reflejan notablemente en el desarrollo de la presencia social se encuentran la actitud y personalidad tanto del estudiante como del tutor, sus habilidades sociales y discursivas, sus estilos de aprendizaje, las estrategias pedagógicas y tecnológicas que se utilicen en el aula y los estímulos en el proceso de la interacción y del aprendizaje. Aquí el rol del tutor juega un papel fundamental.

Se recomienda analizar el diseño instruccional del curso y el objetivo de las actividades colaborativas a fin de que se implemente un poco más la usabilidad de los recursos de la web 2.0, sobre todo el audio y la imagen, como estrategias que pueden mejorar los procesos de comunicación y el ambiente de aprendizaje colaborativo, además de elevar los niveles de motivación entre los miembros de grupo y el tutor.

\section{Referencias bibliográficas}

Aguinaga \& Barragán (2007). La presencia social como elemento minimizador de la deserción en la educación a distancia. Virtual Educa Brasil. Recuperado el 15 de febrero de 2010 en http://e-spacio.uned.es/fez/view.php?pid=bibliuned:19307.

Anderson, T., Rourke, L., Garrison, D. R. \& Archer, W. (2001). Assessing teaching presence in a computer conferencing context. Journal of Asynchronous Learning Networks, 5 (2), 1-17.Aragon, S. R.

Barry Du Vall, Powell, Hodge \& Ellis (2007). Text Messaging to Improve Social Presence in Online Learning. Recuperado el 20 de enero de 2010 en http://www.educause. edu/EDUCAUSE+Quarterly/EDUCAUSEQuarterlyMagazineVolum/TextMessagingtol mproveSocialPr/161829. 
García Cabrero, B., Márquez, L., Bustos, A., Miranda, G. A. \& Espíndola, S. (2008). Análisis de los patrones de interacción y construcción del conocimiento en ambientes de aprendizaje en línea: una estrategia metodológica. Revista Electrónica de Investigación Educativa, 10 (1). Consultado el 24 de febrero de 2010 en: http://redie. uabc.mx/vol10no1/contenido-bustos.html.

Gros, B. \& Silva, J. (2006, Julio). El problema del análisis de las discusiones asincrónicas en el aprendizaje colaborativo mediado. RED (Revista de Educación a Distancia), núm. 16. Consultado el 10 de marzo de 2009 en http://www.um.es/ead/red/16/gros.pdf.

Gunawardena, C. N. (1995). [Social presence theory and implications for interaction and collaborative learning in computer conferences]. International Journal of Educationa Telecommunications, 1(2/3), 147-166.

Gunawardena, C. N., \& Zittle, F. J. (1997). [Social presence as a predictor of satisfaction within a computer-mediated conferencing environment]. The American Journal of Distance Education, 11(3), 8-26.

Universidad Nacional Abierta y a Distancia, UNAD. Recuperado el 10 de febrero de 2010 en http://www.unad.edu.co/index.php?option=com_content\&view=arti cle\&id=33\&ltemid $=206$.

Jerónimo Montes, J A. Hacia las comunidades virtuales de aprendizaje: Aprender para apropiarse de los nuevos medios digitales. Revista Electrónica: Teoría de la Educación. Educación y cultura en la sociedad de la información. Volumen 2009-10 (2). Universidad Nacional Autónoma de México. Consultado en http://campus.usal.es/ teoriaeducacion/ DEFAULT.htm.

KME: Knowledge Management Enviroment. Recuperado el 13 de febrero de 2010 en http://www.jucs.org/jucs_6_5/knowledge_management_and_environmental.

Lowenthal, P. R. Social presence. Regis University, USA. Encyclopedia of distance and online learning. Consultado en http://www.patricklowenthal.com/publications/ socialpresenceEDOLpre-print.pdf.

Pérez, M., Montse Guitert. (Sept 2007). La dimensión social del aprendizaje colaborativo virtual. En catalán, Universitat Oberta de Catalunya, UOC. RED. Revista de Educación a Distancia. Num. 18. consultado el 10 de marzo de 2009 en http://www. um.es/ead/ red/18/perezmateoguitert.pdf.

Richardson. J. C. (2003). Examining social presence in online courses in relation to students' perceived learning and satisfaction. JALN. Volumen 7, Issue 1.

Salazar Ramos, Roberto J. (2008). Lineamientos sobre la estructura de los cursos virtuales en Campus Virtual - Estándar CORE. Universidad Nacional Abierta y a Distancia, UNAD. Bogotá. 
La presencia social en ambientes virtuales de aprendizaje: una estrategia de interacción y construcción del conocimiento, artículo producto de la investigación

.. (2004). El material didáctico en el contexto de la formación a distancia y el sistema de créditos académicos. Universidad Nacional Abierta y a Distancia, UNAD. Bogotá.

......... (2009). Referentes para el desarrollo de contenidos didácticos. Universidad Nacional Abierta y a Distancia, UNAD. Bogotá.

Sampieri, Roberto (2003). Metodología de la Investigación. Mc Graw Hill. 2003. Serrano Núñez, Y. (2005). Assessing faculty's social presence indicators in online courses at the InterAmerican University of Puerto Rico, Bayamón Campus. Consultado el 10 de marzo de 2010 en http://bc.inter.edu/focus/a4_n1/yolanda.pdf.

Short, J., Williams, E. \& Christie, B. (1976). The social psychology of telecommunications. London: John Wiley \& Sons.

Tu, Chih - Hsiung \& Mclsaac, M. S. (2002). [On-line learning migration: From social learning theory to social presence theory in a CMC environment]. Arizona State University. Consultado en www.sciencedirect.com. 